\title{
Systemic anti-commensal response to fungi analyzed by flow cytometry is related to gut mycobiome ecology
}

\author{
Alicia Moreno-Sabater ${ }^{1,2^{*}}$ (D), Gaelle Autaa ${ }^{1}$, Delphine Sterlin ${ }^{1,3,4}$, Amenie Jerbi ${ }^{3}$, Remy Villette ${ }^{1}$, Johanna B. Holm , \\ Christophe Parizot ${ }^{3}$, Sameh Selim ${ }^{6}$, Yaye Senghor ${ }^{2}$, Pascale Ghillani-Dalbin ${ }^{3}$, Claude Bachmeyer ${ }^{7}$, \\ Christophe Hennequin ${ }^{2,8}$, Guy Gorochov ${ }^{1,3}$ and Martin Larsen ${ }^{1}$
}

\begin{abstract}
Background: Interest for the study of gut mycobiota in relation with human health and immune homeostasis has increased in the last years. From this perspective, new tools to study the immune/fungal interface are warranted. Systemic humoral immune responses could reflect the dynamic relationships between gut mycobiota and immunity. Using a novel flow cytometry technology (Fungi-Flow) to determine immunoglobulin (Ig) responses to fungi, we studied the relationships between gut mycobiota and systemic humoral anti-commensal immunity.

Results: The Fungi-Flow method allows a sensitive and specific measurement of systemic lgG responses against 17 commensal and environmental fungi from the two main divisions; Ascomycota and Basidiomycota. IgG responses exhibited a high inter-individual variability. Anti-commensal lgG responses were contrasted with the relative abundance, alpha-diversity, and intra-genus richness of fungal species in gut mycobiota of twenty healthy donors. Categorization of gut mycobiota composition revealed two differentiated fungal ecosystems. Significant difference of anti-Saccharomyces systemic lgG responses were observed in healthy donors stratified according to the fungal ecosystem colonizing their gut. A positive and significant correlation was observed between the variety of IgG responses against fungal commensals and intestinal alpha-diversity. At the level of intra-genus species richness, intense lgG responses were associated with a low intra-genus richness for known pathobionts, but not commensals.

Conclusions: Fungi-Flow allows an easy and reliable measure of personalized humoral responses against commensal fungi. Combining sequencing technology with our novel Fungi-Flow immunological method, we propose that there are at least two defined ecosystems in the human gut mycobiome associated with systemic humoral responses. Fungi-Flow opens new opportunities to improve our knowledge about the impact of mycobiota in humoral anti-commensal immunity and homeostasis.
\end{abstract}

Keywords: Mycobiota, Flow cytometry, Systemic anti-commensal responses, Humoral immunity, Immunoglobulin G, ITS rRNA gene sequencing

\footnotetext{
* Correspondence: alicia.moreno-sabater@sorbonne-universite.fr

'Sorbonne Université, Inserm U1135, Centre d'Immunologie et des Maladies Infectieuses (CIMI-Paris), 75013 Paris, France

${ }^{2}$ Service de Parasitologie-Mycologie AP-HP, Hôpital Saint-Antoine, 75012

Paris, France

Full list of author information is available at the end of the article
}

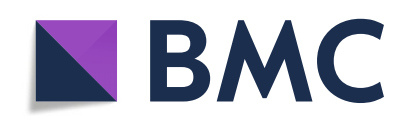

( ) The Author(s). 2020 Open Access This article is licensed under a Creative Commons Attribution 4.0 International License, which permits use, sharing, adaptation, distribution and reproduction in any medium or format, as long as you give appropriate credit to the original author(s) and the source, provide a link to the Creative Commons licence, and indicate if changes were made. The images or other third party material in this article are included in the article's Creative Commons licence, unless indicated otherwise in a credit line to the material. If material is not included in the article's Creative Commons licence and your intended use is not permitted by statutory regulation or exceeds the permitted use, you will need to obtain permission directly from the copyright holder. To view a copy of this licence, visit http://creativecommons.org/licenses/by/4.0/. The Creative Commons Public Domain Dedication waiver (http://creativecommons.org/publicdomain/zero/1.0/) applies to the data made available in this article, unless otherwise stated in a credit line to the data. 


\section{Background}

Advances in high-throughput sequencing and bioinformatics are just beginning to reveal the breadth, depth, and diversity of human mycobiota $[1,2]$. The gut mycobiome is receiving increased attention due to its potential involvement in the etiology of numerous gutassociated diseases. Intestinal fungal dysbiosis may be at the origin of invasive fungal infections due to Candida spp. [3] but it has also been associated with a number of gastrointestinal diseases, chemotherapy-induced enteric disorders, graft-versus-host disease [4], alcoholic hepatitis [5], neurological disease [6], and atopy [7] providing evidence of its ability to influence both local and distal inflammation.

The continuous interaction of the immune system with gut mycobiota is also recognized as important for immune homeostasis, playing a role in immune training, regulation of lymphocyte recirculation and immune tolerance [8-10]. These immunoregulation processes mainly occur at mucosal surfaces, thereby complicating the execution of human studies. Consequently, most of the current understanding of immune-mycobiota interactions, in health or disease, has been acquired from studies using animal models [11-17]. However, conclusions from animal models are hampered by the fact that mouse and human harbor distinct mycobiota ecosystems [18]. Although the use of an animal model is fundamental to decipher immunological mechanisms in situ, the development of new minimally invasive methods is mandatory to corroborate the relevance of this knowledge to human physiopathology.

In this context, flow cytometry methods have been developed to characterize human systemic and local immunoglobulin (Ig) responses to commensal bacteria [19]. This technology has allowed the study of whole bacteria and host Ig relationships from different biological fluids, providing a reliable measure of host exposure to a large range of bacterial species. Using this technology, it has been shown that there is a systemic and mucosal humoral response against commensal bacteria in healthy individuals [20]. Secretory IgA regulates gut microbiota compartmentalization, thereby protecting gut barrier integrity. Systemic anti-commensal IgG is implicated in protection against systemic microbial infections. Systemic anti-microbial IgG responses have also been associated with cancer and autoimmunity, although causality remains to be established in these settings. IgG and IgA have distinct biological roles, but seem to be related since human secretory IgA and systemic IgG converge to target a common fraction of gut microbiota [21].

There is now evidence that systemic anti-commensal responses to fungi play a role in promoting fungal clearance [22-24] and have been associated with immunopathologies characterized by intestinal fungal dysbiosis.
Interestingly, high levels of anti-Saccharomyces cerevisiae antibodies (ASCA), a yeast highly present in the intestinal gut mycobiota, are associated with Crohn's disease, antiphospholipid syndrome, systemic lupus erythematosus, type 1 diabetes mellitus, and rheumatoid arthritis [25]. These findings suggest a role of gut mycobiota in the modulation of anti-commensal systemic responses. However, presently, these responses have been characterized for a reduced number of fungi implicated in human pathology [26]. The main reason being the lack of high-throughput methods covering the high diversity of fungal exposition coming from human mycobiota. Consequently, it remains undiscovered which mycobiota members evoke host immune responses.

Here, we developed a flow cytometry technology for analyzing antibody responses to genotypically and phenotypically different fungi (Fungi-Flow) described in human mycobiota. We exploited the Fungi-Flow method to characterize IgG systemic responses to 17 commensal and environmental fungi in a healthy donor cohort. Combining flow cytometry analysis and mycobiota sequencing, we investigated the relationships between anti-commensal IgG and gut mycobiota diversity.

\section{Materials and methods Fungal biobank}

A fungal biobank was generated including 17 fungal strains from different genera commonly described in human skin, lung, and intestinal mycobiota [27-30]. Fungal reference strains were obtained for Candida albicans (ATCC90028) and Aspergillus fumigatus (ATCC204305). Kluyveromyces lactis, Debaryomyces hansenii, Yarrowia lipolytica, Saccharomyces cerevisiae, Fusarium oxysporum, Acremonium sclerotigenum, Cladosporium cladosporioides, Cyberlidnera fabianii, Cryptococcus neoformans, Penicillium oisonii, Rhodotorula mucilaginosa, Malassezia furfur, Trichosporon inkin, and Mycosphaerella graminicolla and Botrytis circinea were obtained from in vitro culture of stools, skins, and bronchoalveolar lavages from human samples and vegetables. Except for Cryptococcus neoformans, the other fungal isolates belonged to the human flora or vegetables and were not implicated in human pathology. Fungal genus and species were determined by mass spectrometry analysis [31].

\section{Biological samples}

To set up the Fungi-Flow method, IgG and IgA responses were measured in pooled IgG from healthy individuals (Hizentra ${ }^{\circ}$, CSL Behring), human serum from patients suffering from invasive fungal infections $(n=2)$, breast milk $(n=2)$, as well as in bronchoalveolar lavage (BAL) $(n=2)$. To show up the usefulness of the FungiFlow, a cohort of paired plasma and fresh stools $(n=20)$ were collected from healthy donors. Stool and plasma 
collection was carried out as previously described [21]. Oral and written consent were obtained from donors before inclusion in the study. The study protocol was reviewed and approved by the local ethics committee (Comité de Protection des Personnes Ile de France VI, Paris, France). All the biological samples were stored at $-20{ }^{\circ} \mathrm{C}$ until use except the stool samples which were stored at $-80^{\circ} \mathrm{C}$.

\section{Fungi culture conditions}

Fungi were cultured in Sabouraud agar medium (BioRad) between 30 and $35{ }^{\circ} \mathrm{C}$, depending on the fungus studied. To generate budding forms, yeast or spores from filamentous fungi were kept at $4{ }^{\circ} \mathrm{C}$ in RPMI 1640 medium (Gibco) for $2 \mathrm{~h}$ and then cultured at $30{ }^{\circ} \mathrm{C}$ under stirring for $9 \mathrm{~h}$. At the end of the incubation period, cultures were centrifuged at 21,000 g for $20 \mathrm{~min}$. Supernatant was removed and fungi were resuspended in cryopreservation medium A or B. Cryopreservation medium A contains: 1\% (wt/vol) Bacto Peptone (BD Biosciences), $1 \%$ (wt/vol) Bacto yeast extract (BD Biosciences), $0.5 \%$ Glucose (wt/vol) (Sigma Aldrich), and 25\% Glycerol (vol/vol) (Sigma Aldrich) in distilled water. Cryopreservation medium B containing 10\% of Glycerol (vol/vol) (Sigma Aldrich) in PBS (Gibco) was also evaluated. For buddingless fungi, only culture on solid Sabouraud agar medium was realized. Aliquots of $200 \mu \mathrm{l}$ containing $10^{4}-10^{5}$ fungal forms $/ \mu$ l were stored at -80 ${ }^{\circ} \mathrm{C}$ until use.

\section{Quantification of fungi}

To confirm the fungal origin of populations analyzed, fungal stock aliquots $\left(200 \mu \mathrm{l}\right.$ containing $2.10^{5}$ fungal forms $/ \mu \mathrm{l}$ ) were thawed and incubated with $50 \mu \mathrm{l}$ of calcofluor White staining ( $1 \mathrm{~g} / \mathrm{l}$, Sigma-Aldrich) during 15 $\min$ at $4{ }^{\circ} \mathrm{C}$. Then, $400 \mu \mathrm{l}$ of $2 \%$ (wt $/ \mathrm{vol}$ ) paraformaldehyde solution (ThermoFisher) was added to fix samples for $20 \mathrm{~min}$ at $4{ }^{\circ} \mathrm{C}$. After 10 -min 21,000 g centrifugation, supernatant was removed and pellet resuspended in the initial volume. Then, $25 \mu \mathrm{l}$ of fungal suspension were mixed with $25 \mu$ of flow count fluorospheres (Beckman Coulter) in $500 \mu \mathrm{l}$ of PBS. Acquisition of microbeads and fungi was carried out using FACSCanto II flow cytometer (BD) and FACS Diva (BD) software.

\section{Analysis of sporulated and budding fungal forms}

Sorting of C. albicans and A. fumigatus using a forward and side scatter variation gating strategy were performed on a 2-laser two-way fluorescence activated cell sorter (S3 cell sorter; Bio-Rad Laboratories, Hercules, California). The cell shape and purity for both fractions were verified by optical microscopy imaging (Leica DM2500).

\section{Fungal immunostaining detected by flow cytometry (Fungi-Flow)}

Total Ig in serum samples were assessed by a nephelometry method (BN II, Siemens Health Care). In order to normalize the IgG or IgA concentration, serums were diluted to $20 \mu \mathrm{g} / \mathrm{ml}$ for IgG measure and $35 \mu \mathrm{g} / \mathrm{ml}$ for IgA measure in PBS (Gibco). Twenty-five microliters of fixed samples containing $2.5 \times 10^{5}$ fungal forms were distributed in a $1.5 \mathrm{ml}$ tube (Eppendorf). Twenty-five microliters of normalized serum were then distributed in each tube and incubated for $20 \mathrm{~min}$ at $4{ }^{\circ} \mathrm{C}$. Two hundred microliters of PBS were then added and samples were centrifuged at $21,000 \mathrm{~g}$ during $10 \mathrm{~min}$. Supernatant was removed and fungi were incubated with a $25 \mu \mathrm{lmix}$ of goat anti-human IgG Alexa Fluor 647 (Jackson ImmunoResearch) or goat anti-human IgA FITC (Jackson Immunoresearch) or the isotype control, goat IgG A647/ FITC (Jackson Immunoresearch), at $4{ }^{\circ} \mathrm{C}$ during $20 \mathrm{~min}$. After one wash with PBS, stained fungi were resuspended in $200 \mu \mathrm{l}$ of PBS. Acquisition of fungi was performed on a FACS Canto II flow cytometer (BD) using the FACS Diva (BD) software. Unstained spores or budding forms were used as negative control to identify background fluorescence. Monoclonal antibodies with irrelevant specificity (anti-TNF- $\alpha$ human IgG1; MSD laboratory) revealed Fc binding. Median fluorescence intensity (MFI) was used to assess the Ig response.

\section{DNA extraction from stool}

DNA extraction was performed as previously described [21]. Briefly, $200 \mathrm{mg}$ of fecal sample was lysed chemically (guanidine thiocyanate and N-lauroyl sarcosine) and mechanically (glass beads) followed by elimination of cell debris by centrifugation and precipitation of genomic DNA. Finally, genomic DNA was RNase treated. DNA concentration and molecular size were estimated by Nanodrop (Thermo Scientific) and agarose gel electrophoresis.

\section{Real-time quantitative PCR}

$16 \mathrm{~S}$ or $18 \mathrm{~S}$ ribosomal RNA gene levels were determined by real-time quantitative polymerase chain reaction (qPCR) using an ABI 7300 Real time PCR system (Applied Biosystems, Foster City, CA). Amplification and detection were carried out in 96-well plates and with TaqMan universal PCR 2X master mix (Applied Biosystems) to quantify total bacteria and fungi. Primers BactQuant and FungiQuant were used [32, 33]. Bacteria: forward: 5'-CCTACGGGDGGCWGCA-3'; reverse: 5' GGACTACHVGGGTMTCTAATC-3'; probe: (6FAM) 5'-CAGCAGCCGCGGTA-3' (MGBNFQ); Fungi: forward: 50-GGRAAACTCACCAGGTCCAG-3'; reverse: 50-GSWCTATCCCCAKCACGA-3; probe: (VIC) 50TGGTGCATGGCCGTT-30 (MGBNFQ). Each reaction was done in duplicate in a final volume of $25 \mu \mathrm{l}$ with 10 
$\mu \mathrm{l}$ of appropriate dilutions of DNA sample (40 ng DNA). Amplifications were performed as follows: $95{ }^{\circ} \mathrm{C}$ for 10 min, to denature DNA and activate Ampli-Taq Gold polymerase, followed by 50 cycles of $95{ }^{\circ} \mathrm{C}$ for $15 \mathrm{~s}$ and $60{ }^{\circ} \mathrm{C}$ for $1 \mathrm{~min}$. The threshold cycle for each sample was determined for $18 \mathrm{~S}$ gene and normalized to the $\mathrm{Ct}$ value of the all bacteria $16 \mathrm{~S}$ ribosomal RNA gene. Data were calculated using the 2-(2(- $\Delta \Delta \mathrm{Ct}))$ method [34].

\section{DNA sequencing}

Sequencing libraries were constructed by amplifying the 18S rRNA gene ITS2 region using the PCR amplification protocol previously described [28]. DNA samples were subjected to PCR amplification using the following primers:

ITS2_FwTag (5'CTTTCCCTACACGACGCTCTTCCGATCTGTGARTCATCGAATCTTT-3') and ITS2 RvTag (5'-GGAGTTCAGACGTGTGCTCTTCCGAT CTGATATGCTTAAGTTCAGCGGGT-3')

Amplification reaction was performed with DNA MolTaq (Molzym, Bremen, Germany) in a total volume of $50 \mu \mathrm{l}$ containing $1 \mu \mathrm{M}$ of each primer and $1 \mu \mathrm{l}$ genomic DNA from extracted stool samples. Cycling conditions were initial denaturation at $94{ }^{\circ} \mathrm{C}$ for $60 \mathrm{~s}, 35$ cycles of denaturation at $94{ }^{\circ} \mathrm{C}$ for $30 \mathrm{~s}$, annealing at $56{ }^{\circ} \mathrm{C}$ for 30 $\mathrm{s}$, and elongation at $72{ }^{\circ} \mathrm{C}$ for $45 \mathrm{~s}$, followed by a final elongation step at $72{ }^{\circ} \mathrm{C}$ for $7 \mathrm{~min}$. PCR amplicon libraries were finally sequenced on a MiSeq Illumina instrument (Genotoul, Toulouse, France) producing $2 \times 300$ bp paired-end reads.

\section{Gene abundance profiling}

Further processing of demultiplexed sequence reads followed the DADA2 workflow for Big Data (https:// benjjneb.github.io/dada2/bigdata.html) [35] and employed $\mathrm{R}$ software (version 3.5.3) and the DADA2 package (v. 1.5.2). Briefly, sequences were quality filtered, trimmed, and assembled. Read length of 240 bases was chosen for hard trimming of forward and reverse reads, because these were the lengths beyond which median quality scores decreased below 20 for the lowestquality library. Then, 240 bases trimming length was also adopted to ensure that paired sequences contained sufficient information for merging (overlapping 3 ' ends). Individual reads were truncated at the base, where a quality score of 2 was observed, and filtered to contain no ambiguous bases. Additionally, the maximum number of expected errors in a read was set to 2. Chimeras from combined runs were removed by the DADA2 protocol. Amplicon sequence variants (ASVs) generated by DADA2 analysis of the quality-filtered sequence data were taxonomically classified using the RDP naïve Bayesian classifier [35] trained with the UNITE general release v10.10.2017 ITS2 gene sequence database (https://unite. ut.ee/sh_files/sh_general_release_10.10.2017.zip). Read counts for ASVs assigned to the same taxonomy were summed for each sample. For the 20 healthy donor stool samples included in our study, we obtained 1,042,760 paired-end reads (average: 45,337 paired-end reads/sample), which was reduced to 583320 paired-end reads with appropriate phylogenetic assignment (average: 25,362 paired-end reads/sample). We finally rarefied our data to 11,872 paired-end reads per sample.

\section{Data analysis}

Flow cytometry data analysis was performed using Flowjo program (version 9.3.2). Mycobiome composition was studied with the Phyloseq $R$ package (version 1.24) and JMP Pro (version 14). Alpha-diversity was assessed with the Shannon diversity index. The distance matrix was subjected to agglomerative hierarchically clustering using Ward's method. To assess the richness of anticommensal IgG responses in our cohort, an index of IgG responses was determined using the Shannon index formula applied to the MFI values. Species and strain richness was measured as the number of different amplicon sequence variants (ASV) in a genus. Statistical analysis was performed with GraphPad Prism (version 6) and JMP Pro (version 14).

\section{Results}

Flow cytometry analysis differentiate sporulated and budding forms of fungi

A striking feature of fungal biology is its large heterogeneity. Fungi can be yeast or molds and throughout their life cycle they have the ability to adopt different phenotypes, such as sporulated, pseudohyphal, or hyphal forms. Furthermore, antigens differ between different phenotypes [36]. In order to ensure that flow cytometry technology is able to cover a large range of fungal antigens and ensure the assessment of the whole fungal antibody repertoire from sporulated and buddings forms, we produced overnight cultures of C. albicans and A. fumigatus (Fig. 1).

Forward and side scatter dot plots allowed differentiating two populations based on cellular size and structure. Both these populations bound Calcofluor White, which is a fluorescent blue dye binding to polysaccharides on chitin and cellulose that are present in cell walls on fungi. Using Fluorescence-Activated Cell Sorting technology, both populations were separated and subsequently analyzed by microscopy. The population characterized by low SSC-A/FSC-A measures corresponded to non-budding yeast for C. albicans or spores for A. fumigatus, whereas the population with high SSCA/FSC-A measures corresponded to budding forms of C. albicans or A. fumigatus. 


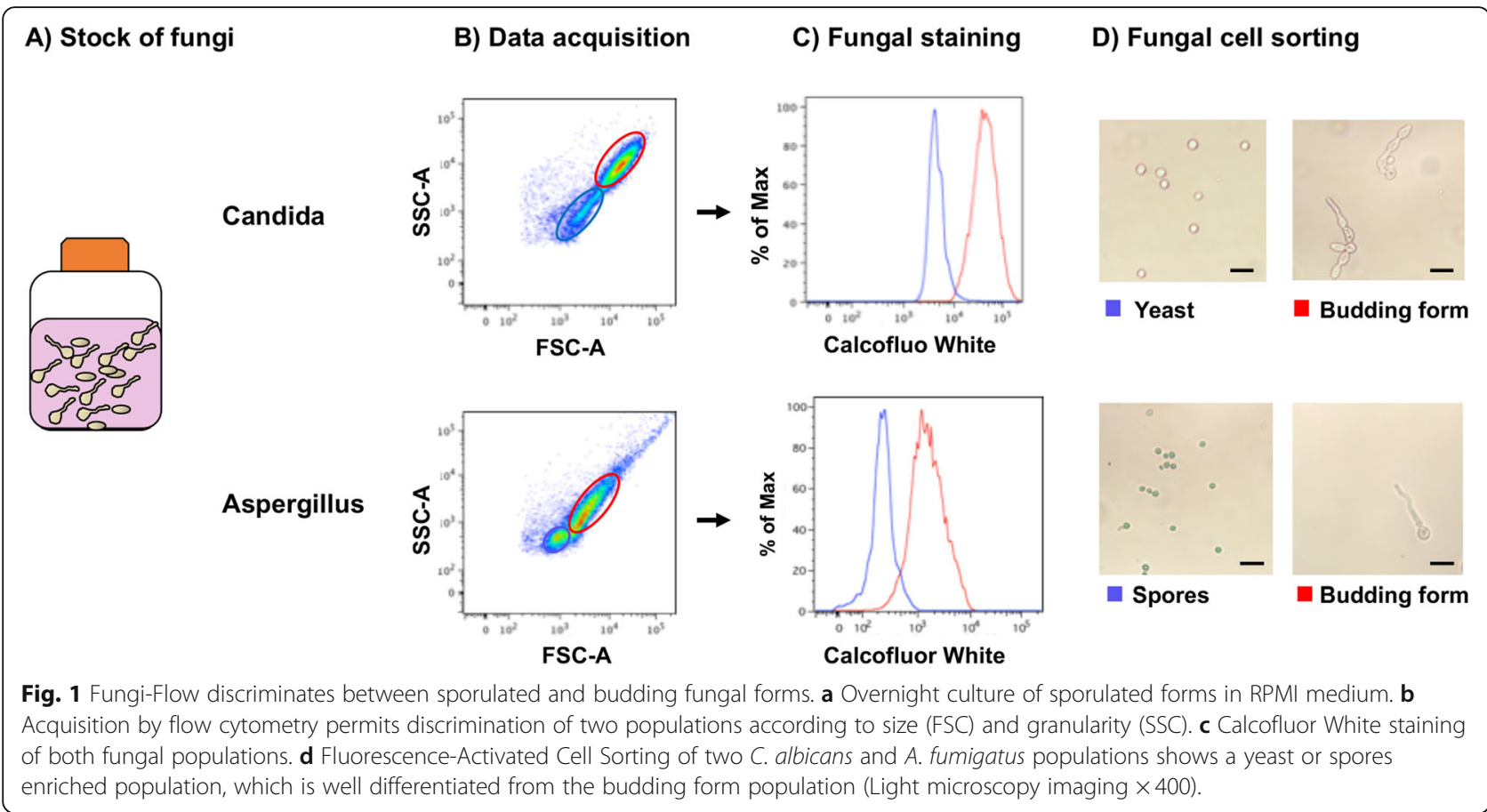

To ensure reproducibility, we sought to produce batches of fungus of a sufficient size to analyze all biospecimens of a given series or cohort. To determine optimal in vitro culture conditions for producing batches of fungal sporulated and budding forms, dose response curves were performed using different initial concentrations of sporulated forms of C. albicans and A fumigatus (Additional file 1: Figure S1). Culture conditions tested for producing batches of C. albicans or A. fumigatus showed that low starting concentrations favored budding form formation. Using $5 \times 10^{6}$ fungal forms $/ \mathrm{ml}$, we obtained both sporulated and budding forms for both fungi. Inoculation of $5 \times$ $10^{6}$ yeasts or spores $/ \mathrm{ml}$ is also suitable for Fusarium and Acremonium (data not shown).

\section{Validation and optimization of analytical specificity and sensitivity}

We sought to set up Fungi-Flow method for anti-fungal IgG and IgA assessment. To determine the optimal IgG concentration to use in the Fungi-Flow method, we realized dose response curves with different fungal concentrations of C. albicans or A. fumigatus and sera from two patients with a confirmed hepatosplenic candidiasis or aspergilloma infection (Fig. 2a, b). Total IgG concentrations tested ranged from 0.1 to $1000 \mu \mathrm{g} / \mathrm{ml}$. We observed the highest IgG-specific responses using $2.5 \times 10^{5}$ fungi per test, for both fungi studied. At this optimal fungal concentration, serial dilutions of total IgG results in sigmoid curves reaching an upper plateau at $20 \mu \mathrm{g} / \mathrm{ml}$. We therefore used serum dilutions of $20 \mu \mathrm{g} / \mathrm{ml}$ total IgG in combination with $2.5 \times 10^{5}$ fungi per test for measuring specific IgG anti-fungal responses. The optimal IgA concentration was also assessed (Additional file 2: Figure S2). Dose response curves using $2.5 \times 10^{5}$ fungi per test resulted in sigmoid curves of MFI reaching an upper plateau at $35 \mu \mathrm{g} / \mathrm{ml}$.

In order to evaluate the specificity of the Fungi-Flow method, we evaluated the specific enrichment of IgG against $C$. albicans and $A$. fumigatus in sera from patients with hepatosplenic candidiasis, aspergilloma, and a healthy donor (Fig. 2c, d). IgG MFIs yielded by the hepatosplenic candidiasis serum against C. albicans were significantly higher than those corresponding to the aspergilloma or healthy donor sera $(p<0.05)$. In contrast, IgG MFIs yielded by the aspergilloma serum against $A$. fumigatus were significantly higher than those yielded by the hepatosplenic candidiasis or healthy donor sera (IgG: $p<0.005$ ). Similar results were obtained when IgA specificity from these sera were evaluated against $C$. albicans and A. fumigatus (Additional file 2: Figure S2). Moreover, using Fungi-Flow method, IgA could be detected in other biological samples such as breast milk and BAL.

We also evaluated the effect of fungal cryopreservation on Ig binding using two different preservation media, described in the materials and methods section (Additional file 3: Figure S3). Similar IgG binding to yeast or budding forms was observed whatever congelation medium was used demonstrating that cryopreservation does not alter Ig binding to fungi forms. We finally chose preservation medium A based on prior experience with clinical isolate preservation. 


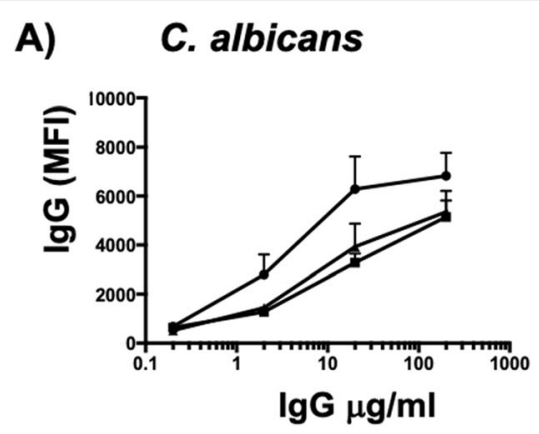

B) A. fumigatus

C)

C. albicans

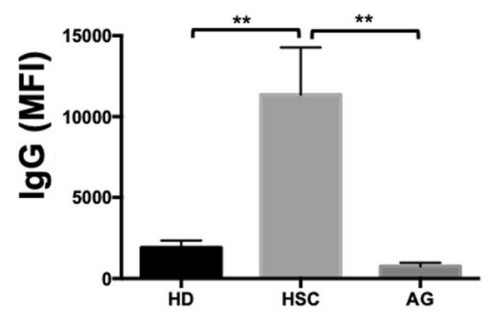

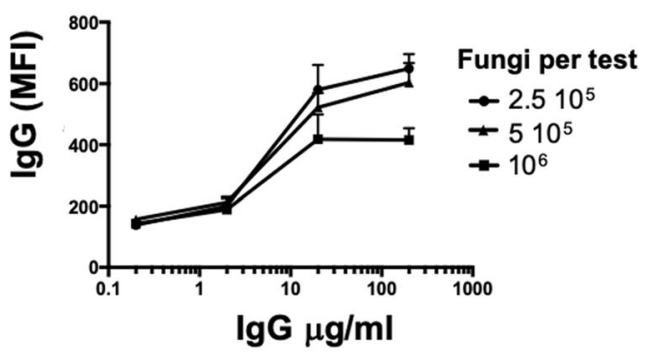

D) A. fumigatus

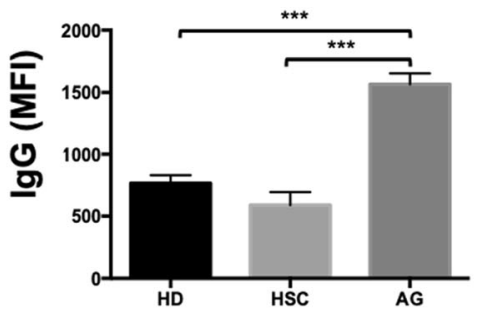

Fig. 2 Fungi-Flow optimal conditions and specificity. a, b Dose response relationship was evaluated by plotting median fluorescence intensity (MFI) obtained for lgG responses at different combinations of C. albicans and A. fumigatus and serum antibody concentrations. Serum was obtained from patients with a confirmed hepatosplenic candidosis (HSC) or aspergilloma (AG). c, d Specificity of antibodies in sera from HSC, AG patients and healthy donor $(H D)$. Results correspond to the mean and standard deviation $(n=3)$. Statistical analysis was performed using Student's $t$ test $\left({ }^{* *} p<0.05,{ }^{* * *} p<0.005\right)$

\section{Fungi-Flow overview}

After determining the optimal assay conditions, we can summarize the Fungi-Flow protocol as shown in Fig. 3. A frozen aliquot containing sporulated and budding fungal forms is thawed, fixed, and then incubated with human fluids to enable specific fungi-antibody interactions (steps $1-3)$. Then, antibody binding is detected by fluorescently labeled secondary antibodies specific for human immunoglobulins, such as IgG or IgA (step 4). Data acquisition is accomplished on a flow cytometer, which enables multiparametric phenotypic assessment, such as size and cellular complexity, biomarkers, such as DNA intercalating agents or chitin binding molecules (Calcofluor White) as well as antibody binding (step 5). Ig histograms are analyzed to

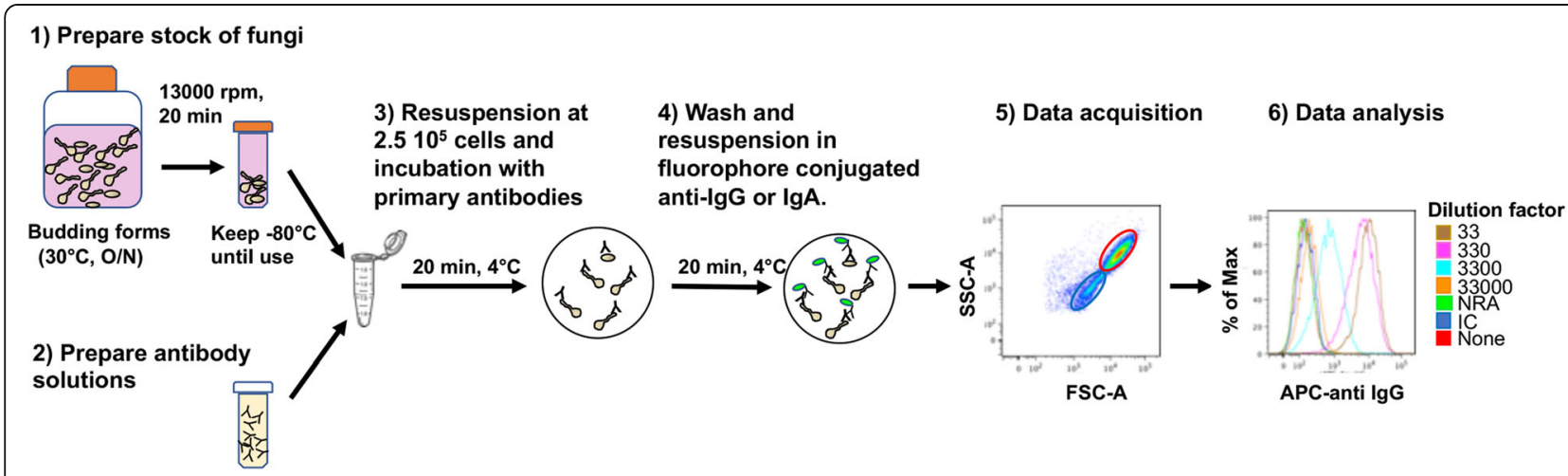

Fig. 3 Overview of the Fungi-Flow protocol. Step 1: Fungal sporulated forms are cultured overnight at $30^{\circ} \mathrm{C}$ to obtain budding forms. They were stored at $-80^{\circ} \mathrm{C}$ until use. Step 2: Antibody samples are diluted to normalized concentrations. Step 3: Fungi are incubated with serum. Step 4: After washing, fungi are incubated with secondary antibodies. Step 5: Data acquisition. Acquisition by flow cytometry permits discrimination of fungi according to size (FSC-A) and granularity (SSC-A). Step 6: Data analysis. Histograms show the median fluorescence intensity (MFI) for yeast forms of C. albicans after incubation with different dilutions of serum from a healthy donor followed by immunostaining with fluorescentlylabeled anti-human IgG antibodies. This protocol can be applied to any culturable fungi. Relevant negative controls must be used to detect unspecific fluorescence. None $=$ unstained fungal forms. NRA non-relevant antibody, IC isotype control 
determine the MFI of the fungal population and quantify the fungal specific antibody binding (step 6). The histogram shows the shift in anti-fungal IgG binding resulting from serial serum dilutions. Fungi-Flow should always be conducted using appropriate positive controls, e.g., immunized sera or polyclonal human IgG, and negative controls, such as no primary antibody, isotype controls, and irrelevant primary antibody (e.g., anti-TNF-alpha antibody).

\section{Systemic anti-commensal immunoglobulin response to} fungi in healthy donor

We then evaluated the performances of the Fungi-Flow to determine the systemic anti-commensal IgG responses in
20 healthy donors. To cover the large fungal heterogeneity, 15 fungi from different genera of the two main divisions Ascomycota and Basidiomycota and commonly described in human skin, lung, and gut mycobiota were chosen [27-29, 37, 38] (Fig. 4). Specific IgG responses were measured against the sporulated forms (Fig. 4a) and for 4 of them equally against the budding forms (Fig. 4b). IgG responses exhibited a high inter-individual variability for a determined genus and this trend was observed for the fifteen different genera studied from the two main divisions. Similarly, anti-fungal IgG responses exhibited inter-taxonomic variability even for closely related taxons, e.g., in the Saccharomycetales order, IgG responses against

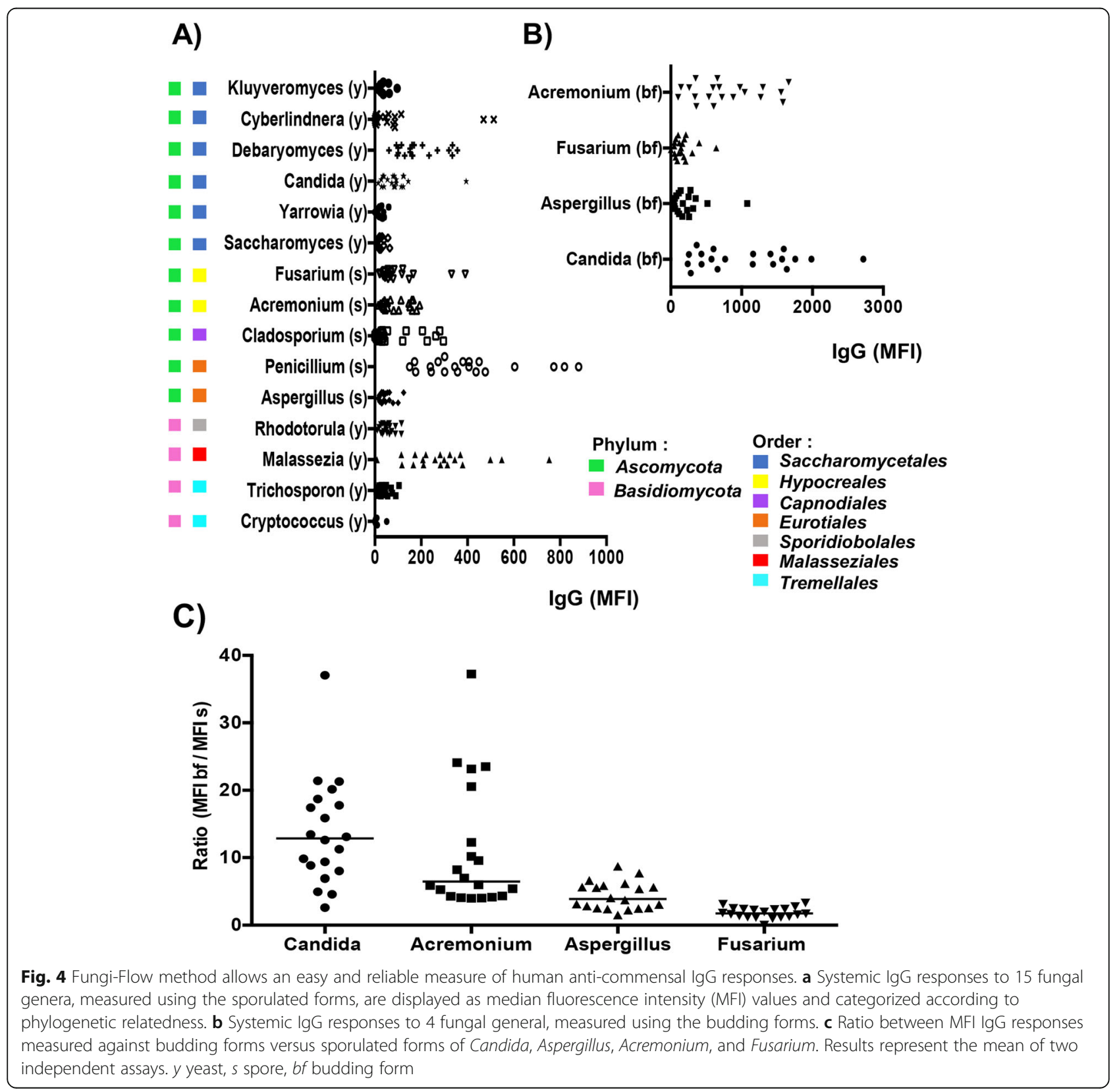


Candida and Debaryomyces were significantly higher than those for Saccharomyces or Kluyveromyces. In the Eurotiales order, Penicillium showed higher responses than Aspergillus. In the Basidiomycota division, Malassezia from the Malasseziales order showed the highest IgG response, whereas Cryptococcus from the Tremellales order showed the lowest. Concerning the anti-commensal response against the budding and invasive fungal forms, the highest responses were observed for Candida and Acremonium, whereas the lowest were for Fusarium and Aspergillus (Fig. 4b). These results confirm that the Fungi-Flow allows an easy determination of systemic anti-commensal responses to a large panel of fungi.

Interestingly, we also noted differences in specific IgG responses between the sporulated or the budding form of a fungus (Fig. 4c). Serum IgG responses against the sporulated and budding forms were correlated but they were not proportional. Thus, for Candida and Acremonium, the IgG ratio between both forms ranged from 2 to 40, whereas the ratio for Aspergillus and Fusarium ranged from 2 to 6 .

\section{Systemic anti-commensal response to fungi is connected to gut mycobiota ecology}

Intestinal mycobiota is an important source of fungal antigens that can elicit systemic IgG anti-commensal responses in animal models [16]. We therefore decided to study the relationships between gut mycobiome composition and the IgG systemic anti-commensal immunity determined using the Fungi-Flow method.

To this aim, global fungal load was determined by qPCR and we characterized the intestinal mycobiota in our healthy cohort of 20 individuals by ITS2 amplicon sequencing analysis. Phylogenetic entities, amplicon sequence variants (AVS), were identified and their relative abundance determined for each donor stool. The most abundant fungal genus in the gut mycobiota was Saccharomyces, followed by Debaryomyces, Candida, Cyberlindnera, and Malassezia, similar to previous observations in healthy individuals [38] (Additional file 4: Figure S4A).

Using the Fungi-Flow methods, IgG responses were analyzed for the 10 more abundant fungi (Additional file 4: Figure S4B). IgG responses for Saccharomyces, Debaryomyces, Candida, Cyberlindnera, Malassezia, Penicillium, and Yarrowia had been determined in the previous section. We then completed this analysis by evaluating the IgG responses against Mycosphaerella, Botrytis, and Trametes. While the IgG responses could be determined against the spore forms for the first two fungi, the lack of in vitro sporulation for the Trametes isolate prevented us from assessing the IgG response against this fungus. Thereafter, the correlation between the fungal relative abundance in gut and the intensity of the systemic response was evaluated. A significant correlation was observed for Saccharomyces $(r=0.51, p$ $=0.0201$ ) suggesting that lower relative abundance of Saccharomyces in gut mycobiota is associated with an increase of ASCA responses.

We further conducted hierarchical clustering of our cohort using the relative abundance for the fifty more represented fungi (Fig. 5). Indeed, two clusters were obtained showing different intestinal fungal composition (Fig. 5a), which were named ecosystem 1 and 2. Compared with the ecosystem 1 , ecosystem 2 was characterized by a significant decrease in Saccharomyces relative abundance and the presence of new genera, such as Cyberlindnera (Fig. 5b). No significant differences were observed in terms of fungal load between both groups (Additional file 5: Figure S5). It is presently unknown if colonization by a given ecosystems is associated with a particular systemic anti-commensal response. We therefore measured the IgG responses to fungi in healthy donors stratified according to their gut mycobiota ecosystem (Fig. 5c). Significantly different ASCA responses were observed between healthy donors harboring the ecosystem 1 and 2, with higher ASCA responses in healthy donors with lower Saccharomyces abundance (ecosystem 2). No correlation was observed between ASCA responses and the relative abundance or antibodies response to other studied fungi (data not shown).

\section{Systemic anti-commensal responses to fungi are related to gut mycobiota diversity}

Subsequently, we hypothesized that gut mycobiota alpha diversity could impacts systemic responses to fungi. To explore this hypothesis, for each healthy donor, we determined an IgG response index using IgG response from fungi described in the previous section and those detected fungi in our healthy population mycobiota and available in our biobank (Cladosporium, Kluyveromyces, Cryptococcus, and Aspergillus) (Fig. 6). Consequently, IgG responses from Fusarium, Acremonium, Rhodotorula, and Thrichosporon were not included in this analysis.

The relationship between the IgG response index and the alpha-diversity of the intestinal mycobiome for each healthy donor showed a significant positive correlation, $r=0.61, p=0.004$. Further analysis revealed significant differences of both alpha diversity and anti-fungal immune diversity between the ecosystems 1 and 2 (Fig. 6b, c). Indeed, a low intestinal diversity was associated with low IgG response index in ecosystem 1, whereas higher diversity observed in the ecosystem 2 was associated with higher IgG response index. 


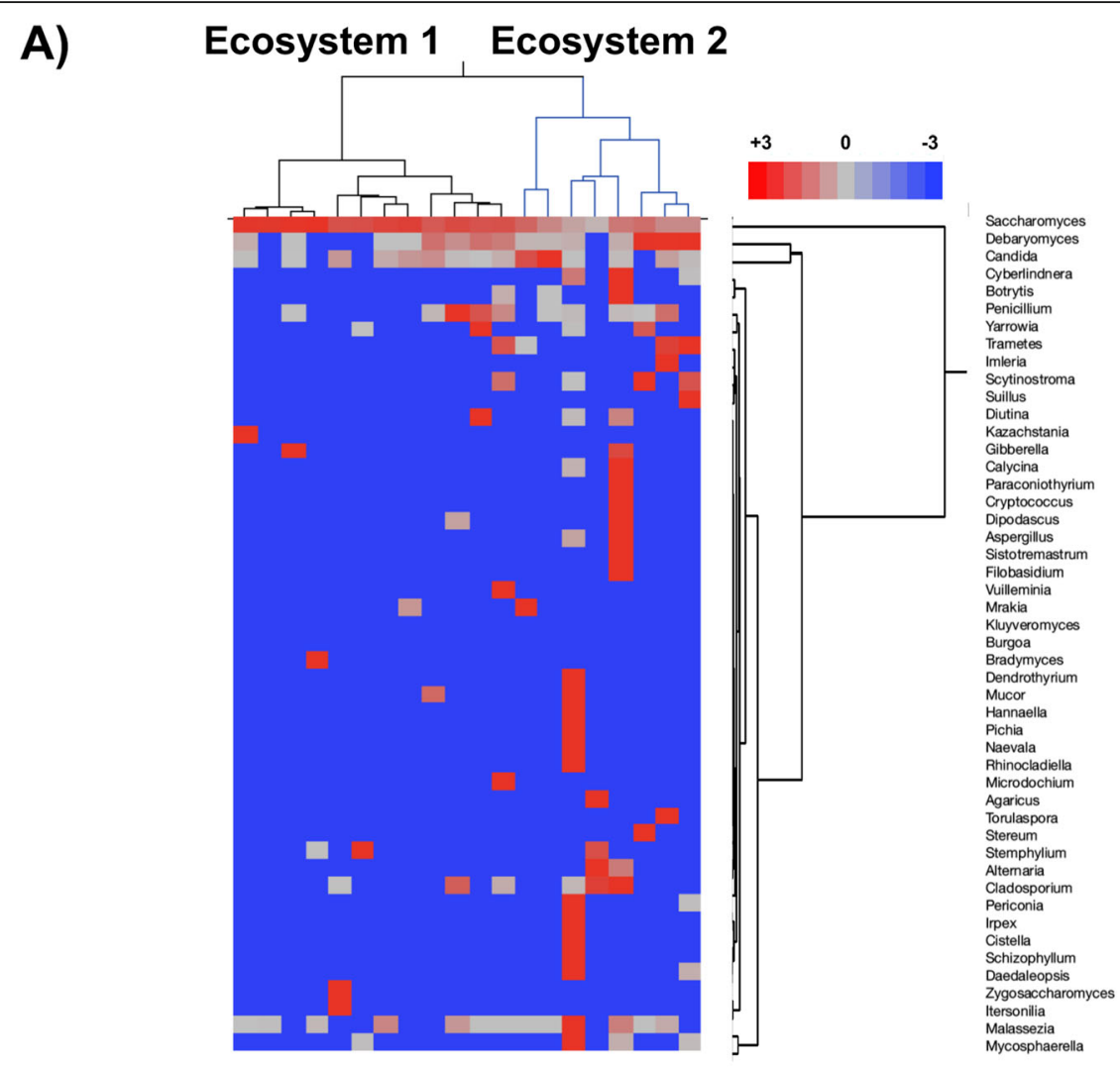

B)

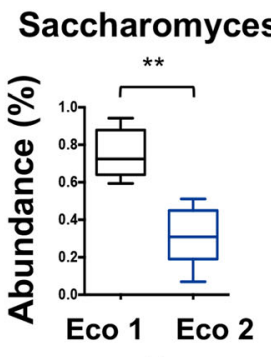

C)

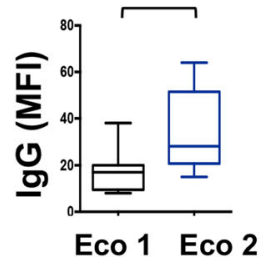

Debaryomyces

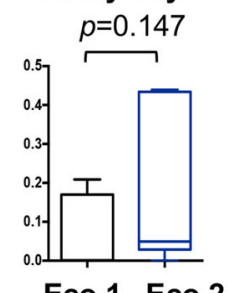

Eco 1 Eco 2

$p=0.925$

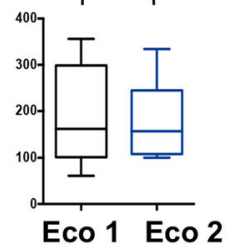

Candida

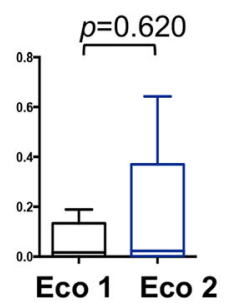

$p=0.112$

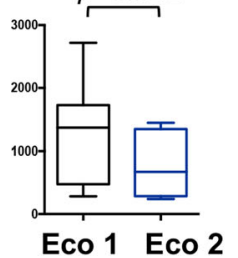

\section{Cyberlindnera}
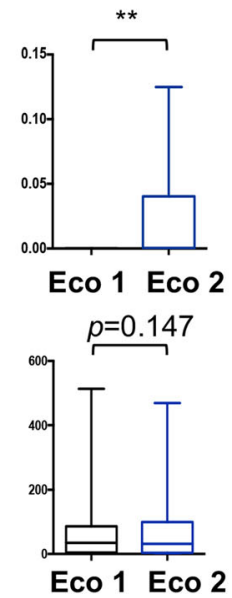

Fig. 5 Gut fungal ecosystems in healthy donors have an impact in systemic anti-commensal response. a Hieratical clustering of the 50 most abundant species in gut mycobiome of healthy donors. This analysis revealed that our healthy population could be shared in two clusters showing two well-differentiated ecosystems (Eco). b Comparison of relative abundances between both ecosystems. Differences were mainly driven by the more abundant fungi, Saccharomyces but also by less abundant fungi such as Cyberlindnera. c Comparison of anti-commensal IgG responses between both ecosystems. Statistical analysis was performed using Mann-Whitney test $\left(^{* *} p<0.05\right)$

Systemic IgG responses are inversely associated with intra-genus gut richness

We also studied the relationship between systemic immunity and the intestinal intra-genus species or strains richness, measured as the number of different ASV belonging to a given genus (Fig. 7). We focused our analysis on two fungi with pathobiont properties, Candida and Malassezia, and three commensal fungi Saccharomyces, Debaryomyces, and Cyberlindnera. For Candida, the relationship between intra-genus richness and the intensity of the anti- 


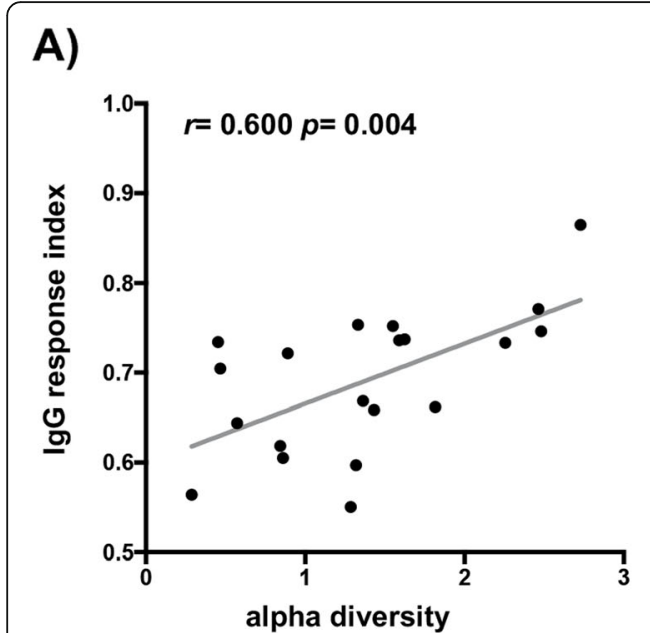

B)

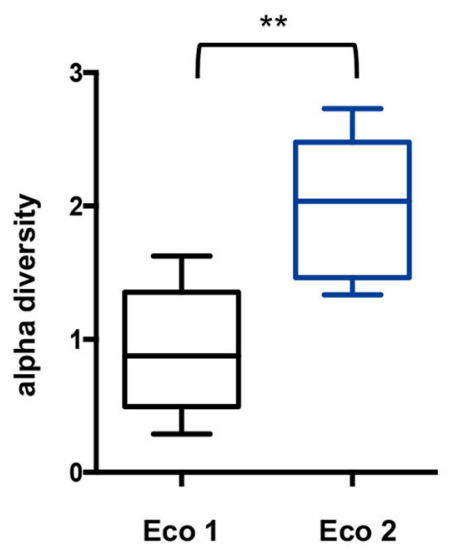

C)

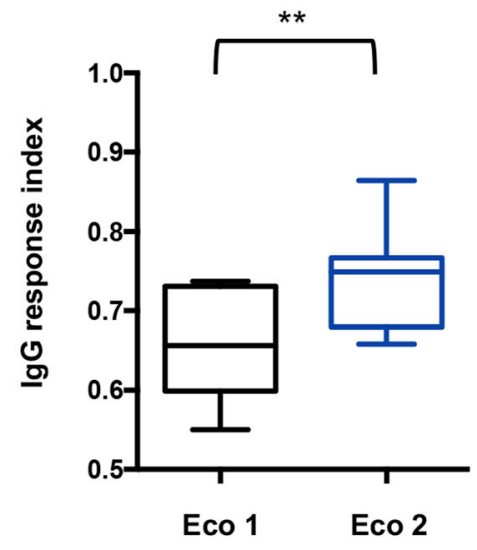

Fig. 6 Diversity of the systemic anti-commensal lgG response is associated with alpha diversity of gut mycobiome in healthy donors. a Analysis of gut mycobiome diversity and its association with the IgG response index observed healthy donors. IgG response index was calculated using the Shannon index formula applied to the MFI values obtained for Saccharomyces, Debaryomyces, Candida, Cyberlindnera, Malassezia, Mycosphaerella, Penicillium, Botrytis, Yarrowia, Cladosporium, Kluyveromyces, Cryptococcus, and Aspergillus. b, c Analysis of alpha-diversity from gut mycobiome and IgG response index in healthy donors harboring different ecosystems. Statistical analysis was performed using Mann-Whitney test $\left({ }^{* *} p<0.05\right)$

commensal IgG responses to Candida was analyzed for the sporulated and budding forms. Systemic IgG responses against commensal pathobionts were significantly higher in those healthy donors having low intra-genus richness (Fig. 7a). Interestingly, for Candida, this difference was significantly different only when we analyzed the IgG response against the budding form, but not against the yeast form. In contrast, no significant difference was observed for the intensity of IgG responses when the intra-genus richness was analyzed for commensal fungi (Fig. 7b).

\section{Discussion}

In this work, a new flow cytometry technology was developed to determine humoral responses against a large range of fungal genera covering the two main Phyla, Ascomycota and Basidiomycota. Our results shown that Fungi-Flow technology allows the assessment of systemic humoral response against 15 different fungal genera commonly described in human mycobiota and 2 specific fungal genera detected in the gut mycobiota of healthy donors included in this study. For 4 of those fungal genera, Fungi-Flow also allows the analysis of IgG responses against their sporulated and budding fungal forms increasing the pipeline of antigens analyzed. It also involves that Fungi-Flow technology is limited to spore forming fungus, which can be grown in vitro. Although not exhaustive, the 17 genera analyzed in the present study cover the most abundant fungal genera described
A)

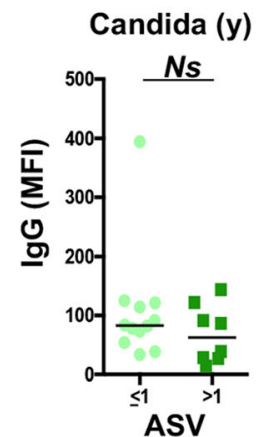

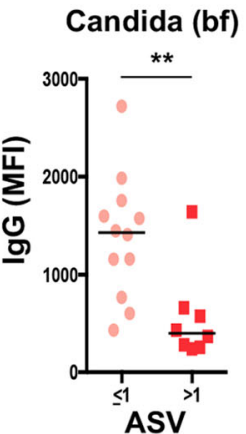

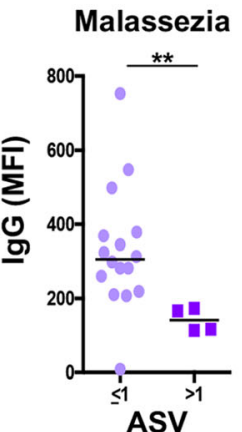

B)

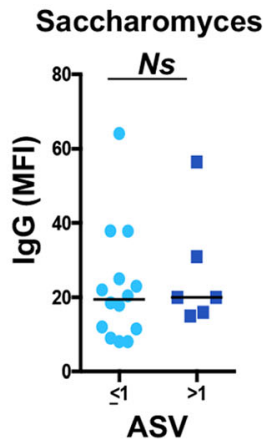

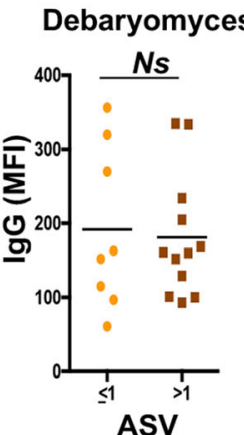

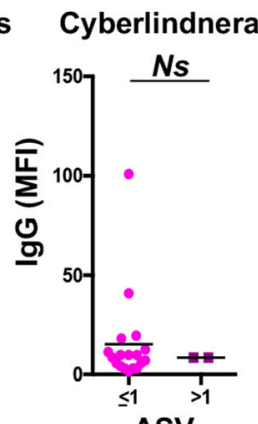

Fig. 7 Relationship between systemic anti-commensal responses and intestinal species or strains richness. IgG response in donors harboring gut a pathobionts or $\mathbf{b}$ commensal fungi with less or more than one different amplicon sequence variant (ASV). Statistical analysis was performed using the Mann-Whitney Wilcoxon test $\left(*^{*} p<0.05\right)$. Ns non-significant difference 
in the human mycobiota $[27-29,37,38]$. This new methodology shows numerous advantages over ELISA and Western blot-based methods, due to its independence from intracellular antigens from lysed fungi and its ability to visualize fungal phenotypes. This technology requires minute quantities of serum and fungal targets thus allowing analysis of fastidious species.

Until now, the assessment of systemic humoral responses to commensal fungi has been hampered by their large variety and heterogeneity and the failure of isolating fungus from stool donors since the vast majority are refractory to cultivation or are rarely obtained in all human samples [1, 39-41]. To study the humoral immunity to commensal bacteria, incubation of healthy donor's serum with isolated gut microbiota have been proposed [21]. However, due to the reduced burden of fungi as compared with bacteria in gut, this method mainly shows the impact of bacteria. As proposed in this study, the indirect assessment of systemic humoral response by incubation of healthy donor's serum with a large variety of fungal strains using flow cytometry technology could move forward the study of immune system and mycobiota interaction as has been demonstrated for bacterial microbiota $[19,42]$.

Using the fungi-flow method, we have characterized for the first time the human anti-commensal IgG response under homeostatic conditions to a broad panel of fungi. IgG responses could be differentiated within a phylogenetic order or even at the genus level, showing a large range of responses inside a population. The intensity of IgG responses varied according to the fungi studied. Intense IgG responses were mainly observed against Penicillium and Malassezia. Penicillium is a common environmental fungus previously described in the lung and gut mycobiome $[29,38]$. It is considered a commensal fungus but its presence in the human environment has been associated with asthma symptoms [43]. Malassezia though present in the intestinal mycobiome is also a skin commensal, involved in skin disorders [30]. A lower level of response was directed to fungi such as Debaryomyces, Fusarium, Acremonium, Cladosporium, and Candida. These fungi are largely represented in diet and environment and it is therefore less clear if they are colonizing commensals or simply in transit. The lowest anti-commensal responses were observed for Kluyveromyces, Cyberlindnera, Yarrowia, Saccharomyces, Rhodotorula, and Trichosporon. All these fungal genera are commensals from human gut or skin and have been rarely implicated in human invasive infections, which could explain the low intensity of anti-commensal response developed. Interestingly, very low IgG responses were detected for Saccharomyces despite high abundance of this fungus in the human gut mycobiome [38]. Altogether, our results show that Fungi-Flow allows the assessment of the anti-commensal IgG profile to a large variety of fungi revealing a fingerprint of fungal exposure in a healthy population.

Characterization of gut mycobiota composition of our cohort showed that healthy individuals can be attributed to one of two clusters representing two well differentiated ecosystems. Differences between the two ecosystems are mainly driven by Saccharomyces abundance and alpha-diversity. Combining the anti-commensal systemic humoral response with gut mycobiota abundance, we highlighted a significant and inverse correlation between the intensity of ASCA responses and Saccharomyces abundances in healthy donors' gut. This observation provides a link between the gut ecosystems and the systemic response. The immunological mechanisms implicated in the ASCA response have not been elucidated. ASCA epitope is an oligomannose expressed in Saccharomyces cerevisiae, but also in other yeasts from the diet such as Debaryomyces [44] or Kluyveromyces [45] suggesting that diet can be a source of antigenic stimulation. ASCA epitope is also expressed in C. albicans, highly present in human gut mycobiota [46]. Fungal dysbiosis with an increase in Candida relative abundance has been associated with ASCA response in different human pathologies $[5,28]$. Our study associates for the first time ASCA with gut fungal ecosystem in homeostatic conditions, for which fungal burden did not differ. No correlation was observed between ASCA response and yeast abundance, other than Saccharomyces, suggesting that others factors play a role in the antigenic stimulation of the ASCA responses observed in a healthy population.

Analysis of mycobiota alpha-diversity and the variability of IgG response revealed a positive and significant correlation suggesting that not only Saccharomyces genus but also the remaining studied fungal genera detected in the mycobiota of our healthy donor have an impact on the anti-commensal response. Indeed, healthy donors harboring a highly diverse ecosystem 2 developed anti-commensal systemic responses significantly different to those observed in healthy donors harboring a lower diverse ecosystem. Finally, this study also revealed a relationship between the anti-commensal response and richness of fungal species or strains within a genus showing that intense IgG responses to pathobionts, such as Malassezia and Candida (for the budding and invasive form), were significantly associated with lower intragenus richness. Species within the same genus with different virulent capabilities or dominance of a more virulent species in the same genera may be responsible for the lowest inter-genus diversity and the intense immune response. In the present study, healthy donors with lower intra-genus richness (ASV $\leq 2)$ were mainly colonized by $C$. albicans and $M$. restricta. Evidences from 
in vitro studies and animal models proposed that the human immune system has learnt to differentiate fungal antigens from Candida hypha and yeast, tolerating those from the sporulated form but reacting to hyphae antigens associated with invasion [47-49]. Our results confirmed these observations in humans and let us to hypothesize that immunoregulation processes might have an impact on gut fungal fitness.

Altogether, these results let us to propose that in healthy donors' intestinal mycobiota influence antifungal antibody responses. Whereas most of the present findings concerning gut mycobiota composition agree with the existence of a core mycobiome [38], a high degree of inter- and intra-volunteer variability has been observed. In the present study, combining the results obtained from immunological and phylogenetic methods, we bring new evidence supporting the hypothesis that different fungal ecosystems exist and that they are associated with a distinct immunological fingerprint.

\section{Conclusions}

Fungi-Flow method allows an easy and reliable measure of personalized humoral responses against commensal fungi. Combining sequencing technology with our novel Fungi-Flow immunological method, we propose that there are ecosystems in the human gut mycobiome associated with systemic humoral responses. Fungi-Flow will improve our knowledge concerning the role of IgG in immune and mycobiome homeostasis or as a marker to characterize immune responses related to autoimmune diseases. This new method could also represent an important advance in the diagnosis of emerging fungal infections for which there are presently no diagnostic methods $[50,51]$

\section{Supplementary information}

Supplementary information accompanies this paper at https://doi.org/10. 1186/s40168-020-00924-8.

Additional file 1: Figure S1. Fungus budding in vitro conditions. A) Side scatter (SSC) and forward scatter (FSC) structural analysis of sporulated and budding forms of $C$. albicans and $A$. fumigatus after overnight $(\mathrm{O} / \mathrm{N})$ culture using different starting concentrations of yeast or spores $/ \mathrm{ml}$. B) Percentage of yeast and budding forms for each condition tested after $\mathrm{O} / \mathrm{N}$ culture.

Additional file 2: Figure S2. Fungi-Flow optimal conditions and specificity for IgA assessment. A-B) Dose response relationship was evaluated by plotting median fluorescence intensity (MFI) obtained for IgA responses at different concentrations of $C$. albicans and A. fumigatus and serum antibody concentrations. Serum was obtained from patients with a confirmed hepatosplenic candidosis (HSC) or aspergilloma (AG). C-D) Specificity of IgA in sera from HSC, AG patients and healthy donor (HD). Results correspond to the mean and standard deviation $(n=3)$. Statistical analysis was performed using Student's t-test $\left({ }^{*} p<0.5^{* *} p<0.05,{ }^{* * *} p<\right.$ 0.005). E-F) IgA responses from breastmilk and bronchoalveolar lavage (BAL) samples of different donors (green and orange lines respectively) were tested for antibody binding to C. albicans and A. fumigatus.
Controls: None = unstained fungal forms (gray filled histogram), isotype control (black line), IgA deficient serum (blue line).

Additional file 3: Figure S3. Fungal stock cryopreservation. A) Histograms show the effect of two cryopreservation mediums $A$ and $B$ on IgG binding in yeast and buddings forms of C. albicans compared with that obtained with freshly grown fungi. Effects of fungus cryopreservation on IgG binding was evaluated using a serum from a patient suffering from hepatosplenic candidiasis (HSC) and a pool of intravenous lgG (IVIG). The effect of congelation mediums was also measured in the different negative controls used. None = unstained fungal forms. NRA= non-relevant antibody, $I C=$ Isotype control. B) Median fluorescence intensity (MFI) measured for each condition. Cryopreservation medium A contains: 1\% (wt/vol) Bacto Peptone (BD Biosciences), 1\% (wt/vol) Bacto yeast extract (BD Biosciences), 0.5\% Glucose (wt/vol) (Sigma Aldrich) and 25\% Glycerol (vol/vol) (Sigma Aldrich) in distilled water. Cryopreservation medium B contains: 10\% of Glycerol (vol/vol) (Sigma Aldrich) in PBS (GIBCO) was also evaluated.

Additional file 4: Figure S4. Relationship between fungal gut abundance and anti-commensal lgG responses. A) Relative abundance of the fifty most abundant fungal genera in gut of healthy donors. B) Correlation between the fungal relative abundance in gut of healthy donors and the intensity of anti-commensal systemic lgG response for the nine more abundant intestinal fungi. Statistical analysis was performed using Spearman tests. Ns= non-significant difference.

Additional file 5: Figure S5. Fungal burden in healthy donors. 165 or $18 \mathrm{~S}$ ribosomal RNA gene levels were determined by real-time quantitative polymerase chain reaction ( $\mathrm{PPCR}$ ). To avoid confounding factors (e.g. different stool hydration) 185 results were normalized using the bacterial $16 \mathrm{~S}$ gene, as previously described [34]. The relative fungal load was calculated using the 2-(2(- $\Delta \Delta \mathrm{Ct}))$ method. Global fungus level in healthy donors from ecosystem 1 and 2 showed no significant differences.

\section{Abbreviations}

ASCA: Anti-Saccharomyces cerevisiae antibodies; ASV: Amplicon sequence variant; Ig: Immunoglobulins; MFI: Median fluorescence intensity

\section{Acknowledgements}

The authors wish to thank technical personnel of the Parasitology and Mycology Service, Saint Antoine Hospital, Paris France and J.L. Perignon for his helpful comments.

\section{Authors' contributions}

AMS, DS, AJ, CB, YS, SS, and ML collected serum, clinical data, and fungal isolates. AMS and GA prepared fungi biobank with yeast/spores and budding forms. DS and PGD measured IgG and IgA levels in samples. AMS, GA, and CP performed flow cytometric analysis. DS, and ML prepared biospecimens (aliquoting and DNA extraction of stool) and cryopreserved them to generate biobank. AMS, GA, JBH, and ML generated ITS2 phylogenetic data. AMS, RV, and ML performed data mining and biostatistical analysis. AMS, GG, and ML designed the study and prepared the figures. $\mathrm{CB}$ and $\mathrm{CH}$ provided support for the design of the study. AMS, GG, and $M L$ wrote the manuscript. The author(s) read and approved the final manuscript.

\section{Funding}

The study was financed by the following funding bodies: Institut national de la santé et de la recherche médicale (Inserm), Sorbonne Universite Emergence (MycELIA-SU-16-R-EMR-45), Agence Nationale de la Recherche (MetAntibody, ANR-14-CE14-0013) and HDHL-Intimic Era-Net (EarlyFOOD).

\section{Availability of data and materials}

All data generated or analyzed during this study are available.

\section{Competing interests}

The authors declare no competing interests.

\section{Author details}

1'Sorbonne Université, Inserm U1135, Centre d'Immunologie et des Maladies Infectieuses (CIMI-Paris), 75013 Paris, France. ${ }^{2}$ Service de 
Parasitologie-Mycologie AP-HP, Hôpital Saint-Antoine, 75012 Paris, France. ${ }^{3}$ Service d'immunologie, AP-HP, Hôpital Pitié-Salpêtrière, 75013 Paris, France. ${ }^{4}$ Unit of Antibodies in Therapy and Pathology, Institut Pasteur, UMR1222 Inserm, 75015 Paris, France. ${ }^{5}$ Institute for Genome Sciences and Department of Microbiology and Immunology, University of Maryland School of Medicine, Baltimore, MD, USA. ${ }^{6}$ College of Agricultural Sciences AGHYLE Res, Unit. Institut Polytechnique UniLaSalle, 60026 Beauvais, France. ${ }^{7}$ AP-HP, Hôpital Tenon 4, rue de la Chine, 75020 Paris, France. ${ }^{8}$ Centre de Recherche Saint-Antoine, CRSA, AP-HP, Sorbonne Université, Inserm, 75012 Paris, France.

\section{Received: 6 July 2020 Accepted: 15 September 2020} Published online: 15 November 2020

\section{References}

1. Huseyin CE, O'Toole PW, Cotter PD, Scanlan PD. Forgotten fungi-the gut mycobiome in human health and disease. FEMS Microbiol Rev. 2017;41(4): 479-511.

2. Limon JJ, Skalski JH, Underhill DM. Commensal Fungi in Health and Disease. Cell Host Microbe. 2017;22(2):156-65.

3. Pappas PG, Lionakis MS, Arendrup MC, Ostrosky-Zeichner L, Kullberg BJ. Invasive candidiasis. Nat Rev Dis Primers. 2018;4:18026.

4. Mukherjee PK, Sendid B, Hoarau G, Colombel JF, Poulain D, Ghannoum MA. Mycobiota in gastrointestinal diseases. Nat Rev Gastroenterol Hepatol. 2015; 12(2):77-87.

5. Lang S, Duan Y, Liu J, Torralba MG, Kuelbs C, Ventura-Cots $M$, et al. Intestinal fungal dysbiosis and systemic immune response to fungi in patients with alcoholic hepatitis. Hepatology. 2019.

6. Forbes JD, Bernstein CN, Tremlett H, Van Domselaar G, Knox NC. A fungal world: could the gut mycobiome be involved in neurological disease? Front Microbiol. 2018;9:3249.

7. Fujimura KE, Sitarik AR, Havstad S, Lin DL, Levan S, Fadrosh D, et al. Neonatal gut microbiota associates with childhood multisensitized atopy and T cell differentiation. Nat Med. 2016;22(10):1187-91.

8. Wheeler ML, Limon JJ, Underhill DM. Immunity to commensal fungi: detente and disease. Annu Rev Pathol. 2017;12:359-85.

9. $\quad$ lliev ID, Leonardi I. Fungal dysbiosis: immunity and interactions at mucosal barriers. Nat Rev Immunol. 2017;17(10):635-46.

10. Li XV, Leonardi I, Iliev ID. Gut mycobiota in immunity and inflammatory disease. Immunity. 2019;50(6):1365-79.

11. Quintin J, Saeed S, Martens JHA, Giamarellos-Bourboulis EJ, Ifrim DC, Logie $C_{\text {, et }}$ al. Candida albicans infection affords protection against reinfection via functional reprogramming of monocytes. Cell Host Microbe. 2012;12(2):22332.

12. Wagener J, Malireddi RK, Lenardon MD, Köberle M, Vautier S, MacCallum DM, et al. Fungal chitin dampens inflammation through IL-10 induction mediated by NOD2 and TLR9 activation. PLoS Pathog. 2014;10(4):e1004050.

13. De Jesus M, Rodriguez AE, Yagita H, Ostroff GR, Mantis NJ. Sampling of Candida albicans and Candida tropicalis by Langerin-positive dendritic cells in mouse Peyer's patches. Immunol Lett. 2015;168(1):64-72.

14. Li X, Leonardi I, Semon A, Doron I, Gao IH, Putzel GG, et al. Response to fungal dysbiosis by gut-resident CX3CR1+ mononuclear phagocytes aggravates allergic airway disease. Cell Host Microbe. 2018;24(6):847-56 e4.

15. Doron I, Leonardi I, lliev ID. Profound mycobiome differences between segregated mouse colonies do not influence Th17 responses to a newly introduced gut fungal commensal. Fungal Genet Biol. 2019;127:45-9.

16. Leonardi I, Li X, Semon A, Li D, Doron I, Putzel G, et al. CX3CR1+ mononuclear phagocytes control immunity to intestinal fungi. Science. 2018;359(6372):232-6.

17. Huertas B, Prieto D, Pitarch A, Gil C, Pla J, Díez-Orejas R. Serum antibody profile during colonization of the mouse gut by Candida albicans: Relevance for Protection during Systemic Infection. J Proteome Res. 2017; 16(1):335-45.

18. Li J, Chen D, Yu B, He J, Zheng P, Mao X, et al. Fungi in gastrointestinal tracts of human and mice: from community to functions. Microb Ecol. 2018; 75(4):821-9.

19. Moor K, Fadlallah J, Toska A, Sterlin D, Balmer ML, Macpherson AJ, et al. Analysis of bacterial-surface-specific antibodies in body fluids using bacterial flow cytometry. Nat Protoc. 2016;11(8):1531-53.

20. Sterlin D, Fadlallah J, Slack E, Gorochov G. The antibody/microbiota interface in health and disease. Mucosal Immunol. 2019.
21. Fadlallah J, Sterlin D, Fieschi C, Parizot C, Dorgham K, El Kafsi H, et al. Synergistic convergence of microbiota-specific systemic lgG and secretory IgA. J Allergy Clin Immunol. 2019;143(4):1575-85 e4.

22. Casadevall A, Pirofski LA. Immunoglobulins in defense, pathogenesis, and therapy of fungal diseases. Cell Host Microbe. 2012;11(5):447-56.

23. Elluru SR, Kaveri SV, Bayry J. The protective role of immunoglobulins in fungal infections and inflammation. Semin Immunopathol. 2015;37(2): 187-97.

24. van Spriel AB. Novel immunotherapeutic strategies for invasive fungal disease. Curr Drug Targets Cardiovasc Haematol Disord. 2003;3(3):209-17.

25. Rinaldi M, Perricone $R$, Blank M, Perricone $C$, Shoenfeld $Y$. AntiSaccharomyces cerevisiae autoantibodies in autoimmune diseases: from bread baking to autoimmunity. Clin Rev Allergy Immunol. 2013;45(2): 152-61.

26. Richardson M, Page I. Role of serological tests in the diagnosis of mold infections. Curr Fungal Infect Rep. 2018;12(3):127-36.

27. Hallen-Adams HE, Suhr MJ. Fungi in the healthy human gastrointestinal tract. Virulence. 2017;8(3):352-8.

28. Sokol H, Leduca V, Aschard H, Pham HP, Jegou S, Landman C, et al. Fungal microbiota dysbiosis in IBD. Gut. 2017;66(6):1039-48.

29. Nguyen LD, Viscogliosi $E$, Delhaes $L$. The lung mycobiome: an emerging field of the human respiratory microbiome. Front Microbiol. 2015;6:89.

30. Sparber F, LeibundGut-Landmann S. Host Responses to Malassezia spp. in the Mammalian Skin. Front Immunol. 2017;8:1614.

31. Dupont D, Normand AC, Persat F, Hendrickx M, Piarroux R, Wallon M. Comparison of matrix-assisted laser desorption ionization time of flight mass spectrometry (MALDI-TOF MS) systems for the identification of moulds in the routine microbiology laboratory. Clin Microbiol Infect. 2019; 25(7):892-7.

32. Liu CM, Kachur S, Dwan MG, Abraham AG, Aziz M, Hsueh PR, et al. FungiQuant: a broad-coverage fungal quantitative real-time PCR assay. BMC Microbiol. 2012:12:255

33. Liu CM, Aziz M, Kachur S, Hsueh PR, Huang YT, Keim P, et al. BactQuant: an enhanced broad-coverage bacterial quantitative real-time PCR assay. BMC Microbiol. 2012;12:56.

34. Liguori G, Lamas B, Richard ML, Brandi G, da Costa G, Hoffmann TW, et al. Fungal dysbiosis in mucosa-associated microbiota of Crohn's disease patients. J Crohns Colitis. 2016:10(3):296-305.

35. Wang Q, Garrity GM, Tiedje JM, Cole JR. Naive Bayesian classifier for rapid assignment of rRNA sequences into the new bacterial taxonomy. Appl Environ Microbiol. 2007;73(16):5261-7.

36. Erwig LP, Gow NA. Interactions of fungal pathogens with phagocytes. Nat Rev Microbiol. 2016;14(3):163-76.

37. Stehlikova Z, Kostovcik M, Kostovcikova K, Kverka M, Juzlova K, Rob F, et al. Dysbiosis of skin microbiota in psoriatic patients: co-occurrence of fungal and bacterial communities. Front Microbiol. 2019;10:438.

38. Nash AK, Auchtung TA, Wong MC, Smith DP, Gesell JR, Ross MC, et al. The gut mycobiome of the human microbiome project healthy cohort. Microbiome. 2017:5(1):153.

39. Huseyin CE, Rubio RC, O'Sullivan O, Cotter PD, Scanlan PD. The fungal frontier: a comparative analysis of methods used in the study of the human gut mycobiome. Front Microbiol. 2017:8:1432.

40. Chen Y, Chen Z, Guo R, Chen N, Lu H, Huang S, et al. Correlation between gastrointestinal fungi and varying degrees of chronic hepatitis B virus infection. Diagn Microbiol Infect Dis. 2011;70(4): 492-8.

41. Gouba N, Raoult D, Drancourt M. Eukaryote culturomics of the gut reveals new species. PLoS One. 2014;9(9):e106994.

42. Haas A, Zimmermann K, Graw F, Slack E, Rusert P, Ledergerber B, et al. Systemic antibody responses to gut commensal bacteria during chronic HIV-1 infection. Gut. 2011;60(11):1506-19.

43. Caillaud D, Leynaert B, Keirsbulck M, Nadif R, group mAw. Indoor mould exposure, asthma and rhinitis: findings from systematic reviews and recent longitudinal studies. Eur Respir Rev. 2018;27:148

44. Shibata N, Onozawa M, Tadano N, Hinosawa Y, Suzuki A, Ikuta K, et al. Structure and antigenicity of the mannans of Candida famata and Candida saitoana: comparative study with the mannan of Candida guilliermondii. Arch Biochem Biophys. 1996;336(1):49-58.

45. Bilang M, Attioui F, Loppinet V, Michalski JC, Bonaly R. Structure of the phosphopeptidomannans from flocculent and non-flocculent yeast Kluyveromyces lactis. Carbohydr Res. 1996;280(2):303-13. 
46. Standaert-Vitse A, Jouault T, Vandewalle P, Mille C, Seddik M, Sendid B, et al. Candida albicans is an immunogen for anti-Saccharomyces cerevisiae antibody markers of Crohn's disease. Gastroenterology. 2006;130(6):1764-75.

47. Gow NA, van de Veerdonk FL, Brown AJ, Netea MG. Candida albicans morphogenesis and host defence: discriminating invasion from colonization. Nat Rev Microbiol. 2011;10(2):112-22.

48. Sudbery PE. Growth of Candida albicans hyphae. Nat Rev Microbiol. 2011; 9(10):737-48.

49. Witchley JN, Penumetcha P, Abon NV, Woolford CA, Mitchell AP, Noble SM. Candida albicans morphogenesis programs control the balance between gut commensalism and invasive infection. Cell Host Microbe. 2019;25(3): $432-43$ e6.

50. Roilides E. Emerging fungi causing human infection: new or better identified? Clin Microbiol Infect. 2016;22(8):660-1.

51. Lee PP, Lao-Araya M, Yang J, Chan KW, Ma H, Pei LC, et al. Application of flow cytometry in the diagnostics pipeline of primary immunodeficiencies underlying disseminated Talaromyces marneffei infection in HIV-Negative children. Front Immunol. 2019;10:2189.

\section{Publisher's Note}

Springer Nature remains neutral with regard to jurisdictional claims in published maps and institutional affiliations.

- fast, convenient online submission

- thorough peer review by experienced researchers in your field

- rapid publication on acceptance

- support for research data, including large and complex data types

- gold Open Access which fosters wider collaboration and increased citations

- maximum visibility for your research: over $100 \mathrm{M}$ website views per year

At $\mathrm{BMC}$, research is always in progress. 